\section{LESSON 5}

MARGINS:

Left, Pica 20; Elite 30;

Right, moved out of the way.

PAPER: Against the paper guide at 0 on the scale on the left. Turn up 7 single lines.

Check the 'ready-to-type' position: feet, body, fingers and eyes.

Warm up

Tap the keys sharply.

Return the carriage smartly.

Keep your eyes on the copy.

Revise the space bar

o key

Practise the reach until you can type 0 with the I finger without looking down.

Keep either the $\mathrm{j}$ finger or the ; finger in position when typing 0 .

Tap the keys sharply.

Keep your eyes on the copy.

b key

Practise the reach until you can type $b$ with the $f$ finger without looking down.

Keep the asd fingers in position.

Keep your eyes on the copy.

Bounce your right thumb off the space bar.

Consolidation

Check the 'ready-to-type' position. Return the carriage smartly.

Keep your eyes on the copy.

Tap the keys sharply.

Try to type each line in $1 / 2$ minute.

Use the paper release lever when taking the paper out of the machine.

Centre and lock the carriage.

Cover the machine.

UNIT 2

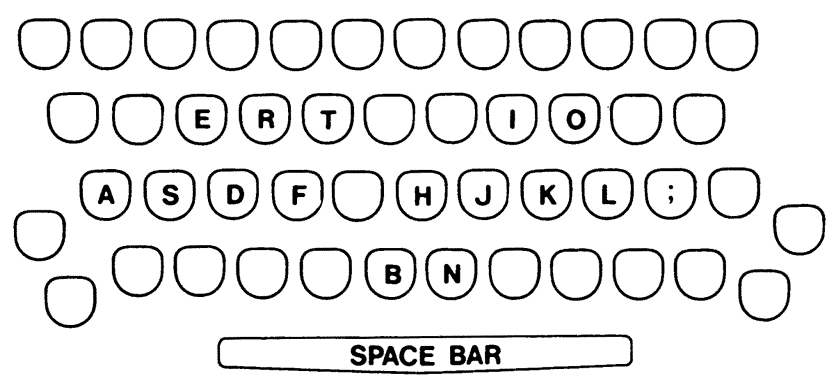

Remember to leave a blank line between sections.

Type the lines indicated by your teacher 3 times each.

1. if he is ill his is a fair sea fish dish

2. the jerk sent the jars hard at the desk;

3. send it; it is flat and red; see the sea

4. the jade is as fine as silk see it shine

Type this line 3 times. Bounce your right thumb off the space bar.

5. $f t j n d e k i j n f r a s ; l a ; j ;$

Type this line as many times as your teacher asks.

6. Ill lol olo loo lol loo olo lolo lol lol

Type the lines indicated by your teacher 3 times each.

7. so so no no do dodo ol old fo for to toe

8. not note lot lot do dole ore ore for for

9. see the old soot; a lost old door; a toe

10. the oil is too hot for the old iron tool

Type this line as many times as your teacher asks.

11. fff fbf fbf bbb fbf bbf fbb fbbf bbf fbf

Type the lines indicated by your teacher 3 times each.

12. be be ba bar eb ebb ob rob ib jib bo bob

13. rib rib be best best band band bank bank

14. a table; a boot; a boat; a bath; a band;

15. both blind rabbits bent the bean bin bar

Type each line 3 times. Try to build up speed each time you repeat a line.

16. it is not fair; and so it is on to do it

17. the earl had both his feet on the brink;

18. the job is hard on one skill as he said;

19. the skiff had half rear bars both sides;

20. the desk still has both ink stands on it

There are 6 lines of type to every inch $(25 \mathrm{~mm})$ down a sheet of typing paper.

There are 70 lines of type down a sheet of A4 paper $(210 \times 297 \mathrm{~mm})$.

There are 35 lines of type down a sheet of $A 5$ paper used sideways $(210 \times 148 \mathrm{~mm})$.

There are 50 lines of type down a sheet of A5 paper $(148 \times 210 \mathrm{~mm})$.

LESSON 5 